\title{
Comparing the SDSS and VLBI quasar and galaxy positions
}

\author{
Sándor Frey*i \\ FÖMI Satellite Geodetic Observatory, Budapest, Hungary \\ MTA Research Group for Physical Geodesy and Geodynamics, Budapest, Hungary \\ E-mail: frey@sgo.fomi.hu
}

\section{Péter Veres}

Physics Department, University College Cork, Cork, Ireland

\section{Krisztián Vida}

Department of Astronomy, Eötvös University, Budapest, Hungary

\begin{abstract}
A direct link between a radio and an optical reference frame can be established by position measurements of common objects. The International Celestial Reference Frame (ICRF) is defined by the positions of 212 compact extragalactic radio sources regularly observed with Very Long Baseline Interferometry (VLBI). At present, space- and ground-based all-sky optical astrometric catalogues do not reach the magnitude limit needed for the detection of the majority of sources studied with VLBI. The HIPPARCOS reference frame in the optical is linked to the ICRF indirectly through relative astrometric measurements of a few radio stars with respect to nearby radio-loud active galactic nuclei (AGNs). In the next decade, sensitive space-based optical astrometry missions (e.g. Gaia) will observe thousands of VLBI-detected quasars, thus providing a direct link between the radio and optical reference frames. Such a link is essential not only for astrometry, but for astrophysical applications as well. The high-resolution structure of the AGNs observed at different electromagnetic wavelengths can only be registered correctly if the coordinates are expressed in consistent systems.

Although the Sloan Digital Sky Survey (SDSS) does not provide high-accuracy astrometric data in the optical, its faint magnitude limit allows us to directly compare the positions of a sample of 735 radio sources (quasars or galaxies). For these objects, ICRF radio positions are available at milli-arcsecond (mas) accuracy or better from the ICRF lists and the VLBA Calibrator Surveys (VCS). We confirm that the SDSS coordinates are in general accurate down to $\sim 60$ mas in both right ascension and declination. We find indications that the optical and radio brightness peaks are not necessarily coincident for some of the objects.
\end{abstract}

8th European VLBI Network Symposium

September 26-29, 2006

Toruń, Poland

\footnotetext{
* Speaker.

${ }^{\dagger}$ This work was supported by the Hungarian Scientific Research Fund (OTKA T046087).
} 


\section{The sample for comparing SDSS and VLBI coordinates}

The imaging catalogue of the most recent, $5^{\text {th }}$ Data Release ${ }^{1}$ (DR5) of the Sloan Digital Sky Survey (SDSS) covers 8000 square degrees of the sky, mainly on the Northern hemisphere and around the Equator. The large sky coverage and the $R \sim 22^{\mathrm{m}}$ limiting magnitude make it possible to identify the counterparts of many radio-loud AGNs that have accurate (mas-level) radio positions available. The source positions in the SDSS are thoroughly calibrated [3] using the best available optical astrometric catalogues.

We compared the ICRF and the VCS ${ }^{2}$ catalogues with the SDSS DR5. We looked for optical cross-identifications of radio sources that have 10 mas formal positional accuracy or better in both right ascension $(\alpha)$ and declination $(\delta)$. (The average values are actually below 1 mas.) This way the radio positions in the sample are at least an order of magnitude more accurate than the optical positions. Among the all-sky set of 3114 ICRF and VCS sources satisfying the above criteria, optical counterparts of 735 were found in the SDSS within a search radius of 300 mas. The majority of our sources are in the range $7^{\mathrm{h}}<\alpha<18^{\mathrm{h}}$ and $-5^{\circ}<\delta<+70^{\circ}$, with a few additional sources evenly distributed in right ascension at $-15^{\circ}<\delta<+15^{\circ}$. (This reflects the SDSS sky coverage.) The distribution of the SDSS-VLBI position differences for the 735 objects - of which 140 are classified as extended (i.e. galaxy) and 595 as point-like (i.e. quasar) in the SDSS - is shown in a histogram in Fig. 1.

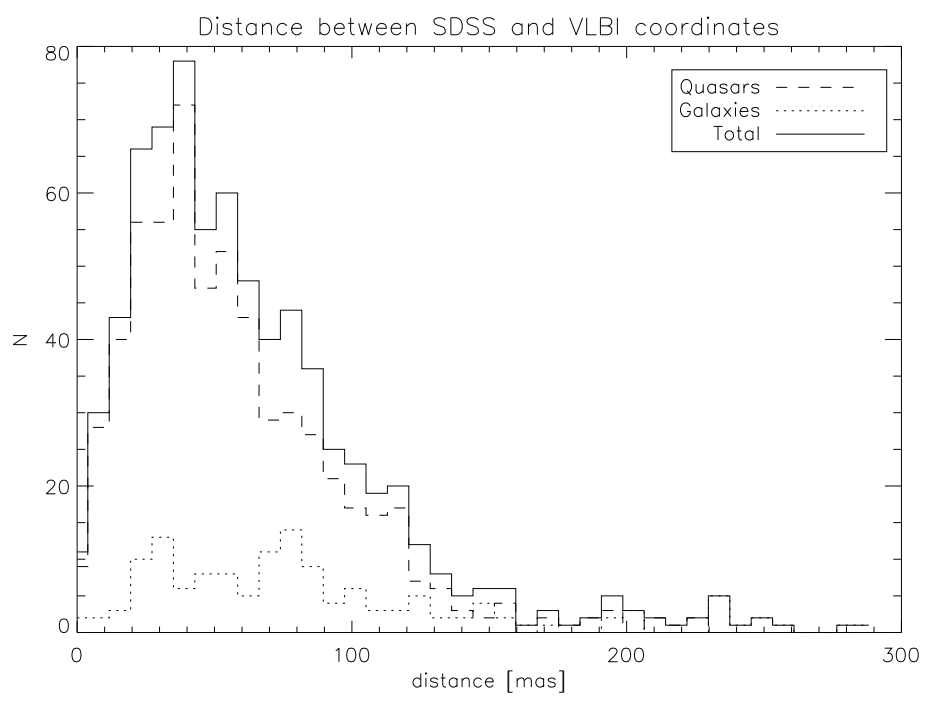

Figure 1: Histogram of optical-VLBI radial position differences for 735 ICRF and/or VCS sources that are also identified in the SDSS DR5 as quasars or galaxies. The distribution is consistent with $\sim 60$ mas $(1 \sigma)$ accuracy in both right ascension and declination in the SDSS.

Our aims were (1) to provide an independent check of the absolute SDSS positional accuracies in general, and (2) to see how the optical-radio position differences depend on object types and certain observing parameters. An earlier version of this study using SDSS DR4 data with 524 matching objects was done in [2].

\footnotetext{
${ }^{1}$ http://www.sdss.org/dr5

${ }^{2}$ http: //www.vlba.nrao.edu/astro/calib
} 


\section{SDSS positional accuracies}

The distribution in Fig. 1 is generally consistent with $\sim 60$ mas positional uncertainty per coordinate, in a good agreement with the values determined for the SDSS [3]. The optical minus radio right ascension $(\Delta \alpha \cos \delta)$ differences have $\sigma_{\Delta \alpha}=58.4$ mas standard deviation and a negligible $\langle\Delta \alpha \cos \delta\rangle=-2.5$ mas mean value. While the declination differences have similar standard deviation $\left(\sigma_{\Delta \delta}=58.5\right.$ mas), there appears an average offset of $\langle\Delta \delta\rangle=13.3$ mas.

For the quasars only, the standard deviations are $\sigma_{\Delta \alpha}=54.6$ mas and $\sigma_{\Delta \delta}=51.7$ mas, and the mean values are $\langle\Delta \alpha \cos \delta\rangle=-0.2$ mas and $\langle\Delta \delta\rangle=13.9$ mas. As expected, for the 140 optically resolved galaxies, the standard deviations are considerably higher in this sub-sample, $\sigma_{\Delta \alpha}=71.9$ mas and $\sigma_{\Delta \delta}=81.8$ mas. The mean optical-VLBI coordinate differences for galaxies are $\langle\Delta \alpha \cos \delta\rangle=-12.1$ mas and $\langle\Delta \delta\rangle=10.7$ mas.

\section{Suspected positional non-coincidences}

The tail of the distribution of coordinate differences in Fig. 1 is worth noting. One may suspect that the outliers seen well beyond the expected radial distance are chance coincidences. To test this hypothesis, we constructed four "false" radio source lists by shifting the right ascension or declination coordinates of all the VLBI sources by a large arbitrary amount $\left( \pm 1^{\circ}\right)$. We then tried to find SDSS optical counterparts within 300 mas radius for these non-existing "objects" (over 12000 in total). Only two chance coincidences were found, suggesting that, unless the corresponding SDSS scans are astrometrically mis-calibrated, most if not all of our outliers (29 AGNs with optical-radio separation larger than 180 mas) in Fig. 1 are real identifications. This result can be considered as a sign of warning. When comparing directly the radio and optical positions, we naturally assume that the optical and radio emission peaks physically coincide. Because the activity of these AGNs is driven by their central supermassive black holes, this assumption seems plausible in general. However, there may exist objects for which it is not necessarily true. For high-accuracy reference frame inter-connections in the future, it is essential to understand this behaviour and to identify the outliers. Such objects should be avoided when the radio and optical reference frames are linked. On the other hand, these AGNs may well be of astrophysical interest.

Although the optical images - at least for the quasars - are unresolved, earlier studies of ICRF radio sources with extended ( $\sim 10$ mas) VLBI structure also found evidence of non-coincidence between the radio and optical centres [1].

\section{References}

[1] D.N. da Silva Neto et al. 2002, A Pattern of Noncoincidence between Radio and Optical Positions of International Celestial Reference Frame Sources, AJ 124, 612

[2] S. Frey, P. Veres \& K. Vida 2006, Celestial positions in radio and optical, Publ. Astron. Dept. Eötvös Univ. 17, 149

[3] J.R. Pier et al. 2003, Astrometric Calibration of the Sloan Digital Sky Survey, AJ 125, 1559 